\title{
How genome editing could be used in the treatment of cardiovascular diseases
}

\author{
Kiran Musunuru*,1 \\ 1'Department of Medicine \& Department of Genetics, Cardiovascular Institute, Perelman School of Medicine at the University of \\ Pennsylvania, Philadelphia, PA 19104, USA \\ * Author for correspondence: kiranmusunuru@gmail.com
}

" $\mathrm{It}$ is clear that while there is significant potential for cardiovascular genome-editing therapies to eventually be translated to patients, there are substantial technical obstacles that remain to be addressed, and discussions about the acceptability of such therapies are just getting underway."

First draft submitted: 8 October 2017; Accepted for publication: 24 October 2017; Published online: 2 February 2018

Keywords: cardiomyopathy • cardiovascular disease • Cas9 • coronary heart disease • CRISPR • embryo • gene therapy $\bullet$ genetics $\bullet$ genome editing $\bullet$ lipids

Genome editing has received widespread attention due to its transformational effects on the practice of biomedical research; the anticipation that it will lead to new treatments and cures of diseases; and the prospect of designer babies who are free of grievous genetic disorders but also possibly bearing advantageous enhancements. With respect to cardiovascular diseases, how far are we away from using genome editing to make a substantial impact on human health?

\section{Knockout versus repair}

The answer is that there is still a long way to go, but the feasibility of cardiovascular therapies has been explored in a variety of proof-of-concept studies. An important consideration is the mechanism of action by which the therapeutic effect would occur. Genome editing entails the use of any of a variety of tools - including zinc-finger nucleases, meganucleases, transcription activator-like effector nucleases (TALENs) and clustered regularly interspaced short palindromic repeats (CRISPR)-CRISPR-associated 9 (Cas9) - that bind to DNA in a sequence-specific fashion and generate double-strand breaks. CRISPR-Cas9 has proven to be particularly adept at inducing double-strand breaks in a variety of genomic loci in a variety of cell types. The more common cellular repair mechanism for doublestrand breaks, occurring in all cells at all times, is nonhomologous end-joining (NHEJ). NHEJ is error-prone and results in insertions and/or deletions of DNA base pairs in a semi-random fashion and with high efficiency. When targeted to the coding sequence of a gene, genome editing can be very effective at knocking out the gene with frameshift mutations, especially with CRISPR-Cas9. When simultaneously targeted to two sites flanking a gene (or part of a gene), NHEJ can efficiently remove the sequence between the two double-strand breaks. In contrast, homology-directed repair (HDR) is restricted to proliferating cells that are in the $S$ and G2 phases of the cell cycle, meaning that HDR is typically far less efficient for genome editing compared with NHEJ. However, unlike NHEJ, HDR permits precision genome editing via the use of a custom-made DNA repair template that carries a desired alteration, whether a point mutation or insertion of a gene cassette. In principle, HDR allows for the therapeutic correction of pathogenic mutations.

\section{Somatic therapy}

The relative efficiencies of NHEJ and HDR dictate the types of cardiovascular therapies that can be undertaken. Disruption or deletion of genes or portions of genes are intrinsically more practicable than gene correction. With the current state of genome-editing technology, treatments to permanently reduce blood lipid levels and, in principle, reduce the lifetime risk of coronary heart disease (CHD) will be more feasible because there are several genes in which naturally occurring loss-of-function mutations substantially reduce lipids and CHD risk but are well 
tolerated in people (i.e., safe with no serious adverse consequences), including PCSK9, ANGPTL3 and APOC3 [14]. NHEJ-mediated disruption of one or more of these genes should have a therapeutic effect. Proof-of-concept studies with PCSK9 in mouse models have demonstrated that CRISPR-Cas9 genome editing of the gene can occur with high efficiency in the adult liver, reducing blood PCSK9 levels by up to $90 \%$ and blood cholesterol levels up to $40 \%$ [5-8]. While there is concern for off-target effects, in other words, mutations occurring at sites other than the target gene, so far the proof-of-concept studies have found little evidence of this phenomenon in vivo, although the currently used techniques are not sensitive enough to detect mutations occurring in less than one in 10,000 cells. Furthermore, serial innovations with CRISPR-Cas9 are progressively reducing its risk of off-target effects [9-11]. Importantly, CRISPR-Cas9 has proven effective in targeting human PCSK9 in authentic human hepatocytes in vivo in a liver-humanized mouse model, suggesting that PCSK9-targeting genome-editing therapies would be similarly effective in live humans [7]. Another example of a potential NHEJ-based cardiovascular therapy is targeting of TTR, which produces transthyretin and can cause cardiac amyloidosis. Although no proof-of-concept $T T R$ genome editing studies have yet been published, RNA interference-based therapy to reduce TTR expression in the liver is proving to be clinically effective in human patients [12].

There is substantial interest in using genome editing to treat genetic disorders that affect the heart, for example, cardiomyopathies and arrhythmic disorders. Most forms of these disorders are caused by dominant mutations. Theoretically these disorders could be treated by HDR-mediated precision genome editing to correct the dominant mutations in cardiomyocytes, but there are currently two major obstacles. First, cardiomyocytes have limited proliferation after birth, which limits the ability to exploit HDR in those cells. Second, proof-of-concept studies suggest that CRISPR-Cas9 is intrinsically less efficient in cardiomyocytes compared with hepatocytes, for unclear reasons [13]. This is true even for unusual disorders for which NHEJ-mediated therapies can be entertained. The best example to date is Duchenne muscular dystrophy, which is typically caused by a frameshift mutation in an exon in the middle of the $D M D$ gene and is often accompanied by dilated cardiomyopathy. Fortuitously, excision of a set of middle exons of the $D M D$ gene can result in skipping of the part of the gene with the frameshift, yielding a smaller form of the dystrophin protein that retains some degree of normal function. Thus, NHEJ at two sites flanking a set of $D M D$ middle exons can be therapeutic (unlike with most genes). A recent study demonstrated that this strategy could ameliorate the cardiomyopathy that manifests in a mouse model of Duchenne muscular dystrophy [14]. Notably, the efficiency of genome editing of $D M D$ was low - apparently reflecting the reduced function of CRISPR-Cas9 in cardiomyocytes - but only a modest restoration of dystrophin function is sufficient to have a phenotypic response. In light of this study, Duchenne muscular dystrophy appears to be the myocardial disorder for which the use of currently available genome-editing technology is most likely to prove fruitful. For other myocardial disorders, new technical advances in genome editing will be needed.

\section{Germline therapy?}

Another important consideration for cardiovascular genome-editing therapies is the timing of treatment for a patient. With respect to lipid-lowering therapies, the clinical benefit likely reflects the cumulative exposure to reduced lipids. In other words, the earlier a genome-editing therapy is given, the more the patient will benefit; a child would benefit more than a young adult, who would benefit more than an elderly adult. A patient might benefit even more if the therapy were given before birth, in utero. Similarly, for myocardial diseases for which HDRmediated therapies would be needed, the therapies would presumably be more effective when cardiomyocytes are still proliferating, in utero. Arguably, the maximum benefit would be obtained if the therapy was administered at the earliest possible time, in the zygote, since every cell in the resulting person would carry the therapeutic change and descendants of the person would carry the therapeutic change as well. This scenario is no longer a theoretical one, in light of a recent report of therapeutic genome editing in human embryos resulting from sperm cells of a man with hypertrophic cardiomyopathy due to a mutation in $M Y B P C 3$ [15]. While the corrected embryos were not intended to be carried to term, the embryos were viable and had the potential to become living people. Thus, a discussion of the social and ethical implications of genome-editing therapies can no longer be avoided.

Although achieving a public consensus on the permissible uses of genome-editing therapies might prove to be untenable, it is instructive to explore what scientists and physicians think and how that, in turn, might influence public opinions. A STAT-Harvard School of Public Health poll found that far more people trusted scientists and physicians to decide whether or not to allow changing the genes of unborn babies $(53 \%)$ than government officials and policy makers (9\%) [16]. A poll of several hundred scientists and physicians at a recent American Heart Association meeting found that there was broad support for adults using a genome-editing therapy to improve their 
health [17]. Indeed, $69 \%$ said they would opt to personally receive a safe, one-shot, genome-editing therapy that permanently reduced the risk of CHD. In contrast, there was little support for adults using a therapy to acquire a desired nonmedical trait such as athletic ability, in other words, an enhancement. Views on human germline genome editing were more restrictive: $61 \%$ felt it would be acceptable for parents to use it to have a healthy biological child when there was no other means to do so (e.g., two parents with sickle cell anemia or cystic fibrosis); $45 \%$ felt it would be acceptable to reduce the risk of their child having a serious medical condition during the child's lifetime (e.g., CHD); and only $2 \%$ felt it would be acceptable to increase the odds of their child having a desired trait.

It is clear that while there is significant potential for cardiovascular genome-editing therapies to eventually be translated to patients, there are substantial technical obstacles that remain to be addressed, and discussions about the acceptability of such therapies are just getting underway.

\section{Financial \& competing interests disclosure}

The author has no relevant affiliations or financial involvement with any organization or entity with a financial interest in or financial conflict with the subject matter or materials discussed in the manuscript. This includes employment, consultancies, honoraria, stock ownership or options, expert testimony, grants or patents received or pending or royalties.

No writing assistance was utilized in the production of this manuscript.

\section{References}

1. Cohen JC, Boerwinkle E, Mosley TH Jr, Hobbs HH. Sequence variations in PCSK9, low LDL, and protection against coronary heart disease. N. Engl. J. Med. 354(12), 1264-1272 (2006).

2. Stitziel NO, Khera AV, Wang X et al. ANGPTL3 deficiency and protection against coronary artery disease. J. Am. Coll. Cardiol. 69(16), 2054-2063 (2017).

3. TG and HDL Working Group of the Exome Sequencing Project, National Heart, Lung, and Blood Institute. Loss-of-function mutations in APOC3, triglycerides, and coronary disease. N. Engl. J. Med. 371(1), 22-31 (2014).

4. Jørgensen AB, Frikke-Schmidt R, Nordestgaard BG, Tybjærg-Hansen A. Loss-of-function mutations in APOC3 and risk of ischemic vascular disease. N. Engl. J. Med. 371(1), 32-41 (2014).

5. Ding Q, Strong A, Patel KM et al. Permanent alteration of PCSK9 with in vivo CRISPR-Cas9 genome editing. Circ. Res. 115(5), 488-492 (2014).

6. Ran FA, Cong L, Yan WX et al. In vivo genome editing using Staphylococcus aureus Cas9. Nature 520(7546), 186-191 (2015).

7. Wang X, Raghavan A, Chen T et al. CRISPR-Cas9 targeting of PCSK9 in human hepatocytes in vivo. Arterioscler. Thromb. Vasc. Biol. 36(5), 783-786 (2016).

8. Chadwick AC, Wang X, Musunuru K. In vivo base editing of PCSK9 (proprotein convertase subtilisin/kexin type 9) as a therapeutic alternative to genome editing. Arterioscler. Thromb. Vasc. Biol. 37(9), 1741-1747 (2017).

9. Slaymaker IM, Gao L, Zetsche B, Scott DA, Yan WX, Zhang F. Rationally engineered Cas9 nucleases with improved specificity. Science 351(6268), 84-88 (2016).

10. Kleinstiver BP, Pattanayak V, Prew MS et al. High-fidelity CRISPR-Cas9 nucleases with no detectable genome-wide off-target effects. Nature 529(7587), 490-495 (2016).

11. Chen JS, Dagdas YS, Kleinstiver BP et al. Enhanced proofreading governs CRISPR-Cas9 targeting accuracy. Nature550(7676), 407-410 (2017).

12. Coelho T, Adams D, Silva A et al. Safety and efficacy of RNAi therapy for transthyretin amyloidosis. N. Engl. J. Med. 369(9), 819-829 (2013).

13. Johansen AK, Molenaar B, Versteeg D et al. Postnatal cardiac gene-editing using CRISPR/Cas9 with AAV9-mediated delivery of short guide RNAs results in mosaic gene disruption. Circ. Res. 550(7676), 407-410 (2017).

14. El Refaey M, Xu L, Gao Y et al. In vivo genome editing restores dystrophin expression and cardiac function in dystrophic mice. Circ. Res. 121(8), 923-929 (2017).

15. Ma H, Marti-Gutierrez N, Park SW et al. Correction of a pathogenic gene mutation in human embryos. Nature 548(7668), 413-419 (2017).

16. STAT. STAT-Harvard poll: Americans say no to 'designer babies'. www.statnews.com/2016/02/11/stat-harvard-poll-gene-editing/

17. Musunuru K, Lagor WR, Miano JM. What do we really think about human germline genome editing, and what does it mean for cardiovascular medicine? Circ. Cardiovasc. Genet. 10(5), e001910 (2017). 
